\title{
1 Pavlovian fear conditioning does not readily occur in rats in naturalistic \\ 2 environments
}

3 Peter R. Zambetti ${ }^{1}$, Bryan P. Schuessler ${ }^{1}$, Bryce E. Lecamp ${ }^{2}$, Andrew Shin ${ }^{3}$, Eun Joo Kim¹, and Jeansok

4 J. $\mathrm{Kim}^{1 *}$

$5 \quad{ }^{1}$ Department of Psychology, University of Washington, Seattle, WA 98195

6 2Undergraduate Program in Neuroscience, University of Washington, Seattle, WA 98195

7 3Undergraduate Program in Human Biology, Stanford University, Stanford, CA 94305

8

$9 \quad$ *Jeansok J. Kim

Email: jeansokk@u.washington.edu

12

13 Author Contributions:

14 P.R.Z, E.J.K., and J.J.K. conceived the study. P.R.Z., B.P.S., B.E.L., and A.S. performed surgery,

15 behavioral experiments and analyses. P.R.Z., B.P.S., E.J.K. and J.J.K. wrote the manuscript. J.J.K.

16 supervised all aspects of the study.

17

18 Keywords:

19 Learning, memory, ethology, anxiety, PTSD, sensitization

20

21 This PDF file includes:

22 Main Text

23 Figures 1 to 3

24 Supplemental Information 


\section{Abstract}

27

Pavlovian fear conditioning, which offers the advantage of simplicity in both the control of conditioned

28 and unconditioned stimuli (CS, US) presentation and the analysis of specific conditioned and

29 unconditioned responses (CR, UR) in a controlled laboratory setting, has been the standard model in

30 basic and translational fear research. Despite 100 years of experiments, the utility of fear conditioning has

31 not been trans-situationally validated in real-life contexts. We thus investigated whether fear conditioning

32 readily occurs and guides the animal's future behavior in an ecologically-relevant environment. To do so,

33 Long-Evans rats foraging for food in an open arena were presented with a tone CS paired with electric

34 shock US to their dorsal neck/body that instinctively elicited escape UR to the safe nest. On subsequent

35 test days, the tone-shock paired animals failed to exhibit fear CR to the CS. In contrast, animals that

36 encountered a realistic agent of danger (a looming artificial owl) paired with a shock, simulating a realistic

37 predatory strike, instantly fled to the nest when presented with a tone for the first time. These results

38 illustrate the survival function and precedence of a nonassociative process, rather than associative

39 conditioning, in life-threatening situations that animals are likely to encounter in nature. 
Main Text

44

\section{Introduction}

Since the time of Watson and Morgan's (1) conception that emotions, such as fear, should be studied as conditioned (acquired) reactions and Watson and Rayner's (2) demonstration that fear can be rapidly learned in 9-month-old "Little Albert," Pavlovian (or classical) fear conditioning has been the paradigm par excellence for studying both normal and abnormal fear behaviors (3-7). Briefly, fear conditioning focuses on how an initially innocuous conditioned stimulus (CS; e.g., auditory, visual, contextual cues), upon pairing with a noxious unconditioned stimulus (US; usually electric shock) that reflexively elicits unconditioned response (UR; namely defensive reactions), becomes capable of eliciting conditioned response (CR; e.g., freezing in rodents, increased skin conductance in humans). A century of fear conditioning research has led to wide-ranging discoveries. In particular, fear conditioning experiments have fundamentally transformed learning theories from the archaic contiguity (or temporal) relationship (8-10) to the modern contingency (or informational) relationship between the CS and US (11-14), revealed detailed neurobiological mechanisms of learning and memory (15-17) and influenced contemporary cognitive behavioral therapy for various anxiety and traumatic-stressor related disorders, such as panic, phobic and posttraumatic stress disorders (18-22).

Despite its utility and appeal, fear conditioning paradigms nonetheless simplify behavioral analyses of fear, ignoring the multitude of actions and decisions that animals and humans utilize to survive the breadth of risky situations in the real world (23-28). Moreover, the prevalent notion that fear conditioning produces adaptive associative fear memory has yet to be ecologically validated. In fact, some researchers have questioned the evolutionary logic underlying fear conditioning; "No owl hoots or whistles 5 seconds before pouncing on a mouse... Nor will the owl give the mouse enough trials for the necessary learning to occur...What keeps animals alive in the wild is that they have very effective innate defensive reactions which occur when they encounter any kind of new or sudden stimulus" (29). Indeed, laboratory rodents exhibit unlearned, instinctive fear responses to advancing artificial terrestrial and aerial predators $(30,31)$, overhead looming stimuli (32), and predator odors (33).

Here, we investigated for the first time whether fear conditioning readily transpires and modifies subsequent behavior of animals in a naturalistic environment. To achieve this, hunger-motivated rats 
71 searching for a food pellet in a large arena—a purposive behavior (34)—were presented with a discrete

72 tone CS followed by a painful US to their dorsal neck/body region by means of chronically implanted

73 subcutaneous wires (Fig. 1A). A dorsal neck/body shock better simulates real predatory strike compared

74 to footshock used in standard fear conditioning studies, as it is unlikely that predators direct their attacks

75 on small prey animal's paws. Additionally, in nature, bodily injuries are normally inflicted by external

76 agents (namely, predators in animals and perpetrators in humans). Thus, other groups of rats were

77 presented with a looming aerial predator (i.e., a lifelike great horned owl) preceded with and without a

78 tone CS and followed by the same US (Fig. 1B-D). A single trial tone-shock, tone-owl, tone/owl-shock and

79 owl-shock training was employed because multiple bodily harm encounters would prove fatal in nature,

80 antithetical to the natural selection of fear conditioning (29). Later, all animals' reactions to the tone cue

81 were examined while foraging for food in the open arena.

82

\section{Results}

\section{Baseline foraging}

Female and male rats were pseudo-randomly assigned to tone-shock ( 8 females, 8 males), owlshock ( 8 females, 8 males), tone/owl-shock ( 6 females, 8 males), and tone-owl (4 females, 4 males) groups and implanted with subcutaneous wires in their dorsal neck/body (Fig. 1A-C). After recovery from the surgery, the rats were trained to exit a nest compartment upon gate opening to procure a sizable $0.5 \mathrm{~g}$ food pellet placed at variable distances in a large, expanding open arena (Fig. 1D, top panel). On the first baseline day, female rats took a significantly longer amount of time to procure the food pellet compared to male rats (Supplementary materials, Fig S1, Baseline day 1). This initial difference in foraging behavior likely represents heightened spatial neophobia (risk-averse to novel environments) in female rats. As rats became familiar with the foraging arena, the latency and duration measures declined across 5 baseline days comparably in both sexes, with no further statistical differences in latencies for pellet procurement. Because there were no reliable sex differences in subsequent fear conditioning dependent variables (Supplementary materials, Fig. S2), the four groups were collapsed across sexes. 


\section{Fear conditioning}

On the training day, all rats first underwent three foraging trials with pellets fixed at the longest distance $(125 \mathrm{~cm})$ to confirm comparable pre-fear conditioning foraging behavior between groups (Fig.

102 2A, Baseline). Afterwards, animals were exposed to a tone-shock, an owl-shock, a tone/owl-shock or a 103 tone-owl pairing in the manner shown in Fig. 1 (Supplementary materials, Movie S1). Those rats

104 presented with the tone CS 5-sec prior to the gate opening (i.e., tone-shock, tone-owl, tone/owl-shock groups) took more time to enter the foraging arena in comparisons to owl-shock animals unexposed to the tone (Fig. 2B, Leave nest latency); this indicates that the tone was a salient cue that animals were

107 attentive to and thus conditionable. Once in the foraging arena, all animals readily advanced toward the pellet and breached the trigger zone $(25 \mathrm{~cm}$ from the pellet) to activate the shock, owl, or owl-shock stimuli (Fig. 2B, Trigger zone latency). In response to the shock, owl, or owl-shock, all rats promptly fled from the foraging arena to the nest (Fig. 2B, Escape latency; Fig. 2D,E, Escape speed). Figure 2C shows representative track plot examples of tone-shock, owl-shock, tone/owl-shock and tone-owl animals

112 successfully procuring the pellet during pre-tone baseline but not during tone conditioning. The fact that 113 the escape latency and running speed were not significantly different between the tone-owl and other 114 groups indicates that the looming owl-induced innate fear sans pain was just as effective in eliciting the

115 flight UR as the painful shock or shock-owl combination. However, inspections of the escape trajectories 116 revealed that the tone-shock and tone-owl groups tended to flee linearly to the nest, whereas the owl-

117 shock and tone/owl-shock groups that experienced a dorsal neck/body shock $100 \mathrm{~ms}$ after the looming 118 owl (mimicking realistic predatory attack) and begun their flight to the nest inclined to escape circuitously 119 (Fig. 2F,H). This was supported by significant group differences in the escape distances (Fig. 2G) and trajectory angles (Fig. 2I), where owl-shock and tone/owl-shock groups traveled longer distances and had

121 higher angle variances, respectively, during their escape routes than tone-shock and tone-owl groups.

\section{$\underline{\text { Context (pre-tone) testing }}$}

On the following day, animals were placed back in the nest and underwent three pre-tone baseline

125 trials (maximum $300 \mathrm{sec}$ to retrieve the food pellet placed at $125 \mathrm{~cm}$ ) to assess whether previous

126 encounters with tone-shock, owl-shock, tone/owl-shock and tone-owl stimuli combinations produced fear 
127 of the arena. As can be seen in Figure 3A, the owl-shock and tone/owl-shock groups took significantly

128 longer latencies to procure the pellet (i.e., the time from gate opening-to-return to nest with the pellet)

129 than the tone-shock and tone-owl groups on the first day of testing. The lengthened times to enter the

130 foraging arena exhibited by owl-shock and tone/owl-shock rats likely reflect inhibitory avoidance resulting

131 from the previous predatory attack experience in the arena (35). In contrast, the fact that the pre-tone test

132 baseline latencies of tone-shock and tone-owl rats (Supplementary materials, Fig. S3) were not reliably

133 different from their baseline latencies from the fear conditioning day (prior to experiencing tone-shock or

134 tone-owl) suggests that contextual fear conditioning failed to transpire in these animals despite their

135 robust escape behavior to tone-shock and tone-owl experiences. Similar patterns of group differences,

136 albeit lesser magnitudes, were observed on the second day of pre-tone baseline trials (Fig. 3C).

$\underline{\text { Tone testing }}$

Immediately after the pre-tone baseline, all groups were subjected to three successive tone test trials

140 (one minute apart). The owl-shock and tone/owl-shock animals continued to take longer latencies to exit

141 the nest compared to tone-shock and tone-owl animals (Fig. 3B, Leave nest latency). Once in the

142 foraging arena, the tone/owl-shock group's latency to approach $25 \mathrm{~cm}$ from the pellet to trigger the tone

143 were marginally but reliably longer than those of tone-shock and tone-owl groups, but not owl-shock

144 group (Fig. 3B, Trigger zone latency). Upon the activation of tone (60 s continuous), the majority of owl-

145 shock and tone/owl-shock animals promptly fled to the nest, thereby significantly increasing the latency to

146 procure the pellet $(60 \mathrm{~s}=$ unsuccessful), whereas the tone-shock and tone-owl animals were largely

147 unaffected by the tone and readily procured the pellet (Fig. 3B, Procure pellet latency). The second day of

148 tone testing yielded similar patterns of group differences (Fig. 3D). Figure 3E shows individual track plots

149 from all animals with the initial number of trial(s) necessitated for successful foraging. Further analyses

150 across tone testing days ( 3 trials/day) showed that the overall success rates of procuring the pellet were

151 significantly lower in owl-shock and tone/owl-shock groups compared to tone-shock and tone-owl groups

152 (Fig. 3F), and that owl-shock and tone/owl-shock animals required extended trials to reliably obtain the

153 pellet (Fig. 3G). Because the temporal interval between the CS and US is well known to be crucial in

154 various types of Pavlovian conditioning, including fear conditioning (36), we examined whether tone fear 
155 conditioning transpired in a specific (optimal) range of interstimulus intervals (ISI) but was masked by

156 non-optimal ISIs. We found no significant correlation between the ISIs and the magnitudes of tone-

157 induced suppression of pellet procurement in tone-shock animals, indicating that tone fear conditioning

158 failed to materialize across varying ISIs of delay conditioning (Fig. 3H). Conversely, in the tone/owl-shock

159 animals, the tone-induced suppression of pellet procurement was uniformly observed across different

160 ISIs, suggesting that the observed fear in these animals may not necessarily reflect Pavlovian

161 conditioning (Fig 3H). These results of delayed tone-shock paired animals failing to show conditioned

162 tone fear and contextual fear suggest that standard fear conditioning does not readily occur in naturalistic

163 environment. Instead, the finding of owl-shock animals displaying robust fear to a novel tone, which the

164 animals never heard before, suggests that non-associative sensitization-like processes play a crucial role

165 in protecting animals in the real world.

166

167

168

169

170

171

172

173

174

175

176

177

178

179

180

181

182

\section{Discussion}

It is generally believed (though never validated) that there is behavioral continuity of Pavlovian fear conditioning from the laboratory to real-life situations, and thus understanding the mechanisms of fear conditioning will have clinical relevance. The present study directly investigated whether fear conditioning readily occurs in naturalistic situations that animals are likely to encounter in their habitats. Standard fear conditioning in rodents takes place in small experimental chambers, and several studies have shown that a single tone CS-footshock US pairing (i.e., delay fear conditioning) reliably produces conditioned freezing in rats and conditioned tachycardia/freezing in mice (37). One-trial delay tone fear conditioning has also been demonstrated in human subjects using a loud white noise US and assessing conditioned skin conductance response (38). However, in the present study, where rats are exhibiting a purposive foraging behavior (34) in a large arena, a delayed pairing of tone CS and dorsal neck/body shock US (tone-shock group) produced virtually no evidence of auditory (and contextual) fear conditioning across a range of CS durations (i.e., ISIs). A similar pairing of tone CS and looming owl (tone-owl group) also failed to produce auditory fear conditioning despite the owl US evoking robust fleeing UR. In contrast, foraging rats that experienced a looming owl and shock pairing (owl-shock group) later exhibited robust fear (escape) behavior to a novel tone presentation. In the tone/owl-shock animals, the escape behavior 
183 was uniformly observed across different ISIs, suggesting that the observed fear to the tone stimulus

184 in this group may not be a Pavlovian response. These findings then point to a nonassociative sensitization (or sensitization-like) process, rather than associative fear conditioning, as playing a vital function in risky (i.e., predatory attack) situations that animals encounter in nature.

The tone CS ( $3 \mathrm{kHz}, 80 \mathrm{~dB}$, ranging 9-86.6 s) and shock US (2.5 mA, $1 \mathrm{~s})$ employed in the present study were effective in eliciting orienting and fleeing responses, respectively, and were presented to animals in the manner (i.e., a delay conditioning) that satisfied the stimuli saliency, intensity, surprising, and temporal contiguity requirements for conditioning (39-41). Then, what can account for one-trial auditory fear conditioning, demonstrated in standard Pavlovian paradigms $(35,37,38,42)$, not emerging

192 in animals that left the safe nest to forage for food in an open arena? It may well be that rats are not 193 biologically predisposed to associate discrete CS and US in natural (complex) environments where 194 competing hunger-driven and fear-driven motivated behaviors are freely expressed. Indeed, in real-life, only a small minority of people experiencing trauma develop posttraumatic stress disorder (PTSD) and even with re-exposure to the same trauma there is low incidence PTSD $(43,44)$. In contrast, standard experimental chambers may be conducive to fear conditioning because they are simple and limit the repertoire of behaviors. The absence of one-trial fear conditioning in a naturalistic setting may be analogous to "The Rat Park Experiment," where rats housed in an enriched environment with plants, trees and social interaction resist drug addiction behavior evident in standard cage-housed rats $(45,46)$.

201 Animals tested in naturalistic paradigms are given choices that do not force their behaviors into 202 dichotomies (i.e., freezing or no freezing; drug craving or no drug craving). Allowing for an expanded 203 behavioral repertoire, while more difficult to study, may thus yield a greater understanding of behaviors 204 and their underlying brain mechanisms.

205 It should also be noted that fear encounters in real life generally occur in the presence of external 206 agents or forms (i.e., predators/conspecifics in animals and assailants/combatants in humans), which is 207 virtually nonexistent in standard Pavlovian fear conditioning paradigms. Thus, the effects of a discernable entity in associative fear learning have never been investigated. By simulating a realistic life-threatening 209 situation, i.e., a looming aerial predator that instinctively elicited flight behavior followed by somatic pain, we found that rats engaged in purposive behavior utilize nonassociative sensitization as their primary 
211 defensive mechanism. The fact that the owl-shock and tone/owl-shock animals exhibited relatively

212 nonlinear, erratic escape trajectories to the nest compared to linear escape trajectories in tone-shock

213 animals (Fig. 2F-I) suggests the intriguing possibility that the same dorsal neck/body shock US may be

214 interpreted as a life-or-death (panic) situation in the presence of an external threat agent versus a mere

215 startling (nociceptive) situation in the absence of an external threat agent. The erratic flight behavior in the

216 presence of a looming owl may represent the penultimate stage of circa-strike, or "life-or-death," behavior

217 within the "predatory imminence continuum" theory (47). Functionally, a sensitized fear system may

218 intensify avoidance behavior, which in turn effectively transposes novel, neutral cues into "false positives"

219 to prioritize survival in natural environment (29). In other words, nonspecific sensitization-based

220 overestimation of danger may be a more prudent course for survival than relatively more specific

221 association-based prediction of danger.

222 Clark Hull (48) has posited that Pavlovian fear conditioning offers biological utility by circumventing a

223 "bad biological economy" of defense reaction always necessitating injury. This prevailing view that

224 ascribes preeminent importance of fear conditioning as the primary defensive mechanism is likely to be a

225 theoretical simplification and provides an incomplete picture of fear, as its function in a natural

226 environment may be rather limited (i.e., lacks face validity). It may well be possible to produce fear

227 conditioning in naturalistic settings with further CS-US trials but then this too would be a bad biological

228 economy as such learning will dramatically reduce biological fitness. It is also important to recognize

229 inconsistencies in the literatures, such as clinical studies that have reported that patients with anxiety

230 disorders, such as phobias, have trouble recalling the particular pairing of the fear event with its aversive

231 consequences $(49,50)$. The increased utilization of naturalistic fear paradigms that simulate dangers that

232 animals and humans encounter in real life will enable us to clarify, update, and revise fear concepts

233 derived largely from fear conditioning studies and in doing so facilitate future progress in the treatment of

234 fear disorders. 


\section{Materials and Methods}

\section{$\underline{\text { Subjects }}$}

Sixty-two Long-Evans rats (3-4 months old; 32 females and 30 males), purchased from CharlesRivers Laboratories, were initially pair-housed by sex for 5-7 days of acclimatization in a climatecontrolled vivarium (accredited by the Association for Assessment and Accreditation of Laboratory Animal Care), with a reversed 12-h light/dark cycle (lights on at 7 PM). After undergoing subcutaneous wire implant surgery (described below), animals were individually housed and placed on a standard food-deprivation schedule with ad lib access to water to gradually reach and maintain $\sim 85 \%$ normal body weight. All experiments were performed during the dark phase of the cycle in strict compliance with the University of Washington Institutional Animal Care and Use Committee guidelines.

\section{Surgery}

Under isoflurane anesthesia, rats were mounted on a stereotaxic instrument (Kopf), and two Tefloncoated stainless-steel wires ( 0.0003 inch bare, 0.0045 inch coated; A-M Systems, Everett, WA) were inserted in the dorsal neck/back region of body. The wire tips were exposed $(\sim 1 \mathrm{~cm})$, bent to a V-shape, and hooked to subcutaneous tissue (36). The other ends of the wires were affixed to a headstage (Plastics One, MS303-120), which was then cemented to the animal's skull embedded with 6 anchoring screws. While still under anesthesia, animals were connected to a shock-apparatus and given a mild shock to observe muscle twitching; 6 rats that showed no reaction to shock were removed from the experiment. Animals were given 4 days of postoperative recovery and were adapted to handling for 5 days before nest habituation.

\section{Foraging Apparatus and Stimuli}

A custom-built foraging arena consisted of a nest ( $69 \mathrm{~cm}$ length $\times 58-66 \mathrm{~cm}$ width $\times 61 \mathrm{~cm}$ height) that opened via an automated sliding gate to reveal a large, expanded foraging area $(208 \mathrm{~cm}$ length $\mathrm{x}$ $66-120 \mathrm{~cm}$ width $\times 61 \mathrm{~cm}$ height) where $0.5 \mathrm{~g}$ food pellets (grain-based; F0171, Bio-Serv) were placed at variable locations (Fig. 1A). The testing room was kept under red light (11 lux foraging area, 2 lux nest area) with constant white noise (72 dB) playing in the background. Prior to placing each 
268 animal, the arena was wiped with $70 \%$ ethanol. The ANY-maze software and Ami interface system

269 (Stoelting) connected to a PC automatically tracked the animal's position in the arena, via a ceiling mounted camera, and triggered the tone, shock and aerial predator stimuli: (i) $3 \mathrm{kHz}, 80 \mathrm{~dB}$ tone CS was

271 produced using Anymaze (Stoelting) and presented through two speakers mounted on the nest-foraging

272 border; (ii) $1 \mathrm{~s}, 2.5 \mathrm{~mA}$ shock US was delivered to the animal's dorsal neck/back region via a headstage

273 tethered to a stimulus-isolator (Bak); (iii) A life-like model owl (31), mounted onto a $92 \mathrm{~cm}$ pneumatic air

274 cylinder (Bimba) at the opposite end of the foraging arena and hidden behind a black curtain, plunged

275 downward towards the rat $(46 \mathrm{~cm} / \mathrm{s})$, then retracted back to it starting position.

276

277

278

279

280

281

282

283

284

285

286

287

288

289

290

291

292

293

294

295

\section{Behavioral Procedure}

Upon reaching and maintaining $85 \%$ normal body weight, animals were transported to the experimental room and underwent series of habituation, baseline, fear conditioning, and testing sessions.

(Habituation days) Animals were placed in the nest scattered with 20 food pellets $(0.5 \mathrm{~g}$, grain-based, Bio-Serv) for $30 \mathrm{~min} /$ day for 2 consecutive days to acclimatize and associate the nest with food consumption.

(Baseline days) After 1 minute in the nest sans food pellets, the gate opened, and the animal was allowed to explore the large foraging arena and find a pellet placed $25 \mathrm{~cm}$ away from the nest (first trial). As soon as the animal took the sizeable $0.5 \mathrm{~g}$ pellet back to the nest, the gate closed. Once the animal finished eating, the second trial with the pellet placed $50 \mathrm{~cm}$ and then the third trial with the pellet placed $75 \mathrm{~cm}$ commenced in the same manner. Animals underwent $3-5$ consecutive baseline days, with the pellet distances gradually extending to 75,100 and $125 \mathrm{~cm}$, and they were also accustomed to tethering beginning on baseline day 3 onward.

(Fear conditioning day) Rats, pseudo-randomly assigned into tone-shock, tone-owl, tone/owl-shock and owl-shock groups (Fig. 1), underwent 3 baseline trials with the pellet placed at $125 \mathrm{~cm}$ from the nest. On the $4^{\text {th }}$ trial, the tone-shock, tone-owl and tone/owl-shock animals were exposed to a tone CS that came on 5 seconds before the gate opened and remained on until they reached the trigger zone $(25 \mathrm{~cm}$ to the pellet). For tone-shock and tone-owl animals, the tone co-terminated with the shock US and the owl looming, respectively. For tone/owl-shock animals, the shock occurred $0.1 \mathrm{~s} \mathrm{sec}$ after the owl looming and 
296 co-terminated with the tone. Two animals in the tone/owl-shock group were excluded because they

297 failed to leave the nest within 2 min. The owl-shock animals were subjected to the same owl looming-

298 shock pairing (as the tone/owl-shock animals) but in the absence of tone. All rats fled to the nest in

299 reaction to the shock and/or looming owl, at which time the gate was closed. After 1 minute in the

300 nest, the animals were placed back into their homecage.

301 (Testing days) All rats underwent 3 baseline trials (a maximum of $300 \mathrm{sec}$ to retrieve the pellet) to

302 assess whether shock and/or looming owl encounter the previous day resulted in the fear of the

303 arena (i.e., contextual fear). Afterwards, animals were presented with the tone cue when they

304 approached the trigger zone (25 $\mathrm{cm}$ to the pellet). The tone played continuously for $60 \mathrm{sec}$, after

305 which the tone test trial ended. Animals underwent 3 tone tests daily until they successfully attained

306 the pellet (i.e., fear extinction).

\section{$\underline{\text { Data Analyses }}$}

309 Statistical analyses were performed using SPSS (IBM, version 19) and R (The R Foundation, 310 version 3.5.3). Body tracking positions were obtained using Deep Lab Cut (51) and analyzed using a 311 self-written script in Python (Python Software Foundation). Animal sample sizes were determined 312 using a power analysis performed by G*Power (G*Power, version 3.0.1, Franz Faul; power=0.95, 313 alpha $=0.05$, effect size $=0.5$, two-tailed). A Levene's test for normality showed significance for the 314 data, thus nonparametric tests were used for analysis. Because there were no significant sex 315 differences in any stages of the experiment after the first day of baseline (Supplementary materials, 316 Fig. S1), data from females and males were pooled together for all analyses (Supplementary 317 materials, Fig. S2). Statistical significance was set at $P<0.05$. Graphs were made using GraphPad 318 Prism (version 8).

319 Data Availability

320 The data that support the findings of this study and the relevant analysis code are available from the 321 Dryad data repository. https://doi.org/10.5061/dryad.76hdr7sxk Reviewer Link: 


\section{Acknowledgments}

We thank Lori A. Zoellner for valuable comments on the manuscript, and Heather Wu for

327 (to J.J.K.).

328 Disclosures

All authors report no biomedical financial interests or potential conflicts of interests.

\section{References}

332 1. Watson JB \& Morgan JJB (1917) Emotional reactions and psychological experimentation. Am J Psychol 28(3):163-174.

3342 2. Watson JB \& Rayner R (1920) Conditioned emotional reactions. J Exp Psycho/ 3(3):1-14.

335 3. LeDoux J (1998) Fear and the brain: where have we been, and where are we going? Biol 336 Psychiatry 44(12):1229-1238.

337 4. Fendt M \& Fanselow MS (1999) The neuroanatomical and neurochemical basis of conditioned 338 fear. Neurosci Biobehav Rev 23(5):743-760.

339 5. Maren S \& Quirk GJ (2004) Neuronal signalling of fear memory. Nat Rev Neurosci 5(11):844-852.

$340 \quad 6 . \quad$ Bouton ME, Mineka S, \& Barlow DH (2001) A modern learning theory perspective on the etiology $341 \quad$ of panic disorder. Psychol Rev 108(1):4-32.

342 7. Kim JJ \& Jung MW (2006) Neural circuits and mechanisms involved in Pavlovian fear conditioning: a critical review. Neurosci Biobehav Rev 30(2):188-202.

344 8. Watson JB (1913) Psychology as the behaviorist views it. Psychological Review 20(2):158-177.

345 9. Pavlov IP (1927) Conditioned Reflexes: An Investigation of the Physiological Activity of the $346 \quad$ Cerebral Cortex (Oxford University Press, London) p 142.

347 10. Guthrie ER (1930) Conditioning as a principle of learning. Psychological Review 37(5):412-428.

348 11. Kamin LJ (1968) Attention-like processes in classical conditioning in Miami symposium on the 349 prediction of behavior, ed Jones MR (University of Miami Press), pp 9-33.

350 12. Rescorla RA (1968) Probability of shock in the presence and absence of CS in fear conditioning. $351 \quad J$ Comp Physiol Psychol 66(1):1-5. 
352 13. Wagner AR, Logan FA, Haberlandt K, \& Price T (1968) Stimulus selection in animal

353 discrimination learning. J Exp Psychol 76(2):171-180.

354 14. rescorla RA \& Wagner AR (1972) A theory of Pavlovian conditioning: variations in the effectiveness of reinforcement and nonreinforcement (Appleton-Century-Crofts, New York) p 37.

15. Josselyn SA \& Tonegawa S (2020) Memory engrams: Recalling the past and imagining the future. Science 367(6473).

16. Tovote P, Fadok JP, \& Luthi A (2015) Neuronal circuits for fear and anxiety. Nat Rev Neurosci 16(6):317-331.

17. Haubensak W, et al. (2010) Genetic dissection of an amygdala microcircuit that gates conditioned

18. Foa EB \& Rothbaum BO (1998) (Guilford Press, New York).

19. Butler AC, Chapman JE, Forman EM, \& Beck AT (2006) The empirical status of cognitivebehavioral therapy: a review of meta-analyses. Clin Psychol Rev 26(1):17-31.

20. Delgado MR, Olsson A, \& Phelps EA (2006) Extending animal models of fear conditioning to humans. Biol Psychol 73(1):39-48.

367 21. Mahan AL \& Ressler KJ (2012) Fear conditioning, synaptic plasticity and the amygdala:

22. Craske MG, et al. (2011) What is an anxiety disorder? Focus 9(3):20.

23. Lima SL \& Dill LM (1990) Behavioral Decisions Made under the Risk of Predation - a Review and Prospectus. Canadian Journal of Zoology 68(4):619-640.

372 24. Bednekoff PA (2007) Foraging in the Face of Danger (University of Chicago Press, Chicago).

$37325 . \quad$ Stephens DW (2008) Decision ecology: foraging and the ecology of animal decision making. Cogn Affect Behav Neurosci 8(4):475-484.

375 26. Beckers T, Krypotos AM, Boddez Y, Effting M, \& Kindt M (2013) What's wrong with fear $376 \quad$ conditioning? Biol Psychol 92(1):90-96.

377 27. Mobbs D \& Kim JJ (2015) Neuroethological studies of fear, anxiety, and risky decision-making in 378 rodents and humans. Curr Opin Behav Sci 5:8-15. 
379 28. Pellman BA \& Kim JJ (2016) What Can Ethobehavioral Studies Tell Us about the Brain's Fear System? Trends Neurosci 39(6):420-431.

381 29. Bolles RC (1970) Species-Specific Defense Reactions and Avoidance Learning. Psychological Review 77(1):32-48.

383 30. Choi JS \& Kim JJ (2010) Amygdala regulates risk of predation in rats foraging in a dynamic fear environment. Proc Natl Acad Sci U S A 107(50):21773-21777.

31. Zambetti PR, Schuessler BP, \& Kim JJ (2019) Sex Differences in Foraging Rats to Naturalistic Aerial Predator Stimuli. iScience 16:442-452.

32. Yilmaz M \& Meister M (2013) Rapid innate defensive responses of mice to looming visual stimuli. Curr Biol 23(20):2011-2015.

33. Papes F, Logan DW, \& Stowers L (2010) The vomeronasal organ mediates interspecies defensive behaviors through detection of protein pheromone homologs. Cell 141(4):692-703.

391 34. Tolman EC (1948) Cognitive maps in rats and men. Psychol Rev 55(4):189-208.

392 35. Wilensky AE, Schafe GE, \& LeDoux JE (2000) The amygdala modulates memory consolidation of fear-motivated inhibitory avoidance learning but not classical fear conditioning. J Neurosci

36. Lee T \& Kim JJ (2004) Differential effects of cerebellar, amygdalar, and hippocampal lesions on

37. Stiedl O \& Spiess J (1997) Effect of tone-dependent fear conditioning on heart rate and behavior of C57BL/6N mice. Behav Neurosci 111(4):703-711.

38. Guimaraes FS, Hellewell J, Hensman R, Wang M, \& Deakin JF (1991) Characterization of a psychophysiological model of classical fear conditioning in healthy volunteers: influence of gender, instruction, personality and placebo. Psychopharmacology (Berl) 104(2):231-236.

402 39. Rescorla RA (1988) Behavioral studies of Pavlovian conditioning. Annu Rev Neurosci 11:329352.

404 40. Thompson RF \& Krupa DJ (1994) Organization of memory traces in the mammalian brain. Annu Rev Neurosci 17:519-549. 
406 41. Fanselow MS \& Wassum KM (2015) The Origins and Organization of Vertebrate Pavlovian Conditioning. Cold Spring Harb Perspect Biol 8(1):a021717.

408 42. Lee HJ, Berger SY, Stiedl O, Spiess J, \& Kim JJ (2001) Post-training injections of catecholaminergic drugs do not modulate fear conditioning in rats and mice. Neurosci Lett 303(2):123-126.

411 43. Palgi Y, Gelkopf M, \& Berger R (2015) The inoculating role of previous exposure to potentially traumatic life events on coping with prolonged exposure to rocket attacks: A lifespan perspective.

414 44. Somer E, et al. (2009) Israeli civilians under heavy bombardment: prediction of the severity of post-traumatic symptoms. Prehosp Disaster Med 24(5):389-394.

416 45. Alexander BK, Beyerstein BL, Hadaway PF, \& Coambs RB (1981) Effect of early and later colony 417 housing on oral ingestion of morphine in rats. Pharmacol Biochem Behav 15(4):571-576.

418 46. Gage SH \& Sumnall HR (2019) Rat Park: How a rat paradise changed the narrative of addiction. $419 \quad$ Addiction 114(5):917-922.

420 47. Fanselow MS \& Lester LS (1988) A functional behavioristic approach to aversively motivated behavior: Predatory imminence as a determinant of the topography of defensive behavior (Lawrence Erlbaum Associates Inc).

423 48. Hull CL (1929) A functional interpretation of the conditioned reflex. Psychological Review $424 \quad 36(6): 498-511$.

425 49. Lazarus AA (1971) Behavior Therapy and Beyond (McGraw-Hill Companies).

426 50. Öhman A \& Mineka S (2001) Fears, phobias, and preparedness: Toward an evolved module of 427 fear and fear learning. Psychological Review 108(3):483-522.

428 51. Mathis A, et al. (2018) DeepLabCut: markerless pose estimation of user-defined body parts with deep learning. Nat Neurosci 21(9):1281-1289. 
A
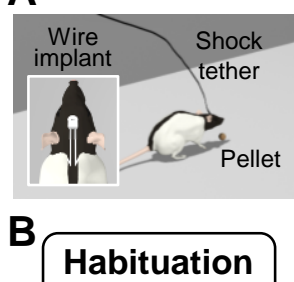

(2 days)

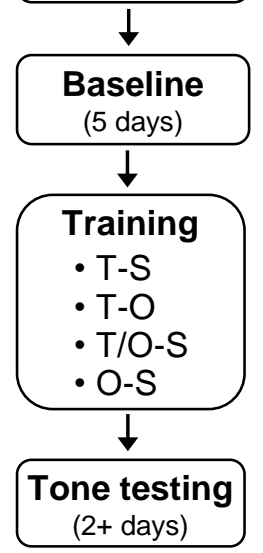

C

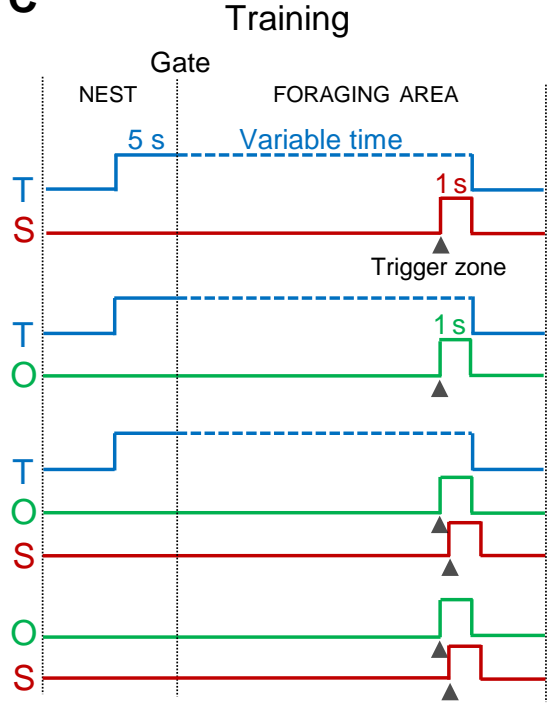

Tone testing

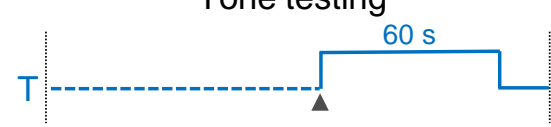

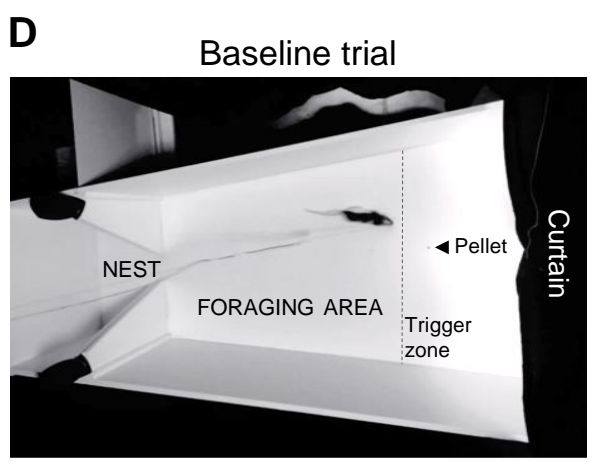

T/O-S trial

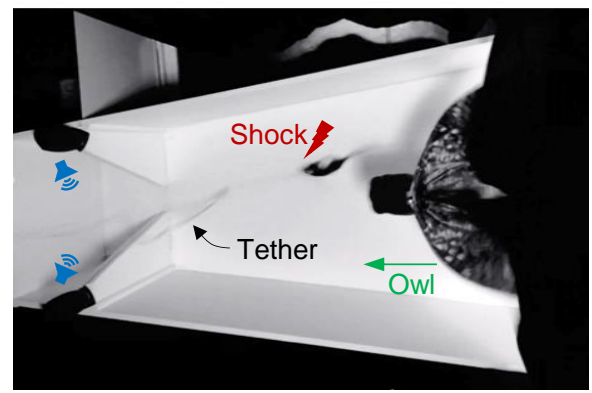

433

434

435

436

437

438

439

440

441

442

443

444

445

446

Fig. 1 Experimental design of fear conditioning in a naturalistic setting. (A) An illustration of a tethered rat foraging for a food pellet in the open arena (inset shows a headstage and placement of subcutaneous shock wires). (B) Timeline of experiment. Habituation: Rats were placed in a closed nest with dispersed food pellets for $30 \mathrm{~min} / \mathrm{day}$. Baseline: Rats were allowed to leave the nest to discover food pellets placed $25-125 \mathrm{~cm}$ (in $25 \mathrm{~cm}$ increments from the nest) in the foraging arena. Training: Animals approaching the pellet location experienced a delayed pairing of tone-shock (T-S), tone-owl (T-O), tone/owl-shock (T/O-S), or owl-shock (O-S). Tone Test: On subsequent days, all rats were placed back in the foraging arena and upon nearing the food pellet, the tone was activated. (C) Schemas of delayed pairings of stimuli. The T-S, T-O and T/O-S (but not O-S) groups were presented with a tone $5 \mathrm{~s}$ before the gate opening that stayed on until the animals were within $25 \mathrm{~cm}$ of the food pellet, at which the tone co-terminated with the triggered shock ( $1 \mathrm{~s})$, owl (1 s) or owl-shock (100 ms interstimulus interval, ISI) stimuli. (D) A representative rat in the foraging arena $(208 \mathrm{~cm}$ length $\times 66-120 \mathrm{~cm}$ expanding width $\times 61$ $\mathrm{cm}$ height) during a baseline trial, where the animal successfully acquires the pellet, and during a T/O-S 
bioRxiv preprint doi: https://doi.org/10.1101/2021.10.20.465116; this version posted October 21, 2021. The copyright holder for this preprint (which was not certified by peer review) is the author/funder, who has granted bioRxiv a license to display the preprint in perpetuity. It is made available under aCC-BY 4.0 International license.

447 trial, where the animal flees from looming owl and shock into the nest $(69 \mathrm{~cm}$ length $\times 58-66 \mathrm{~cm}$ width $\mathrm{x}$

$44861 \mathrm{~cm}$ height).

449

450

451

452

453

454

455

456

457

458

459

460

461

462

463 

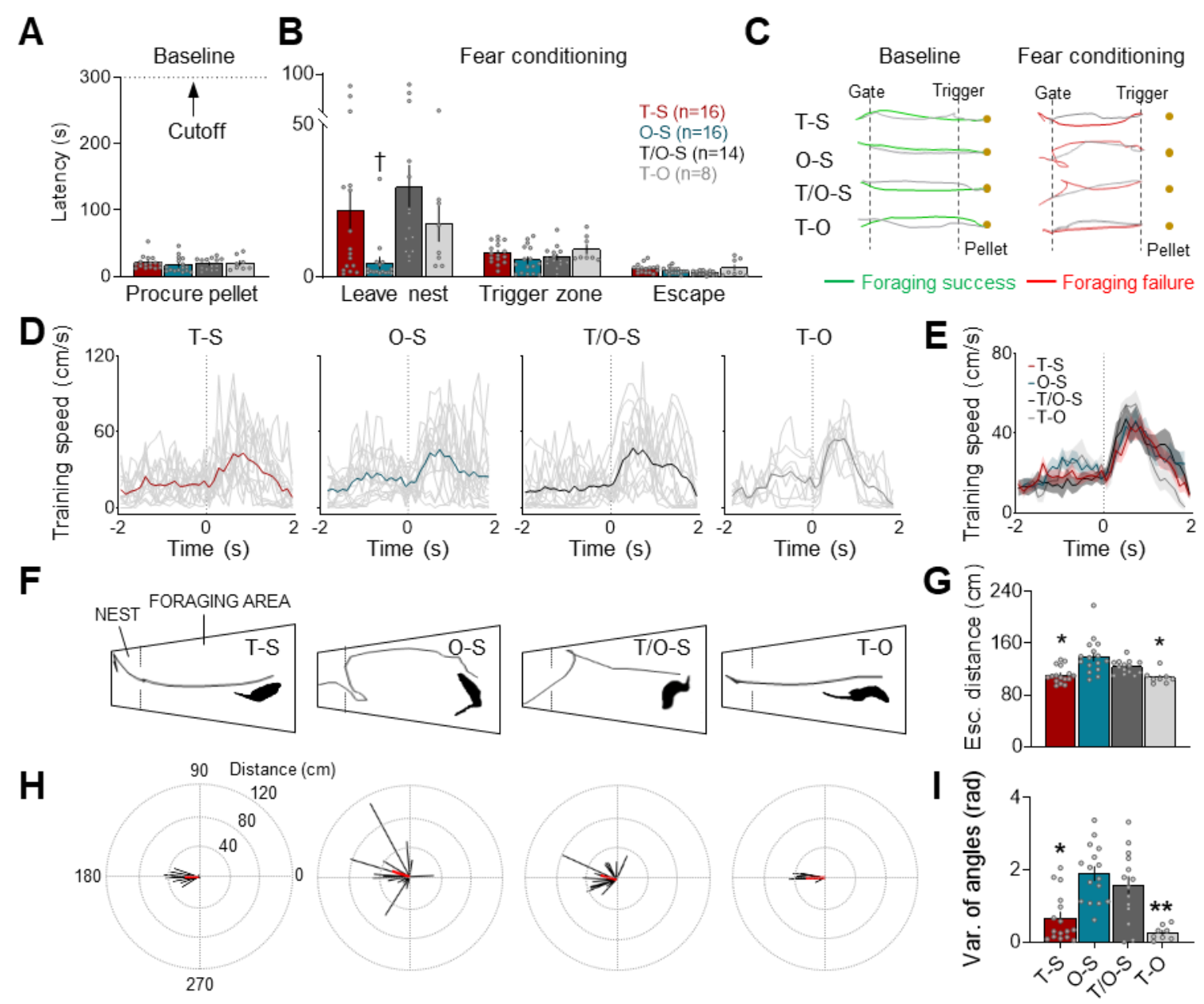

Fig. 2 Foraging and escape behaviors during fear conditioning. (A) Pre-conditioning baseline latencies (mean \pm SEM) to procure food pellets in the foraging arena were equivalent between T-S (red), O-S (blue), T/O-S (dark gray) and T-O (light gray) groups (Kruskal-Wallis, $\mathrm{H}=2.694, p=0.441$ ). (B) During fear conditioning, the T-S, T/O-S and T-O groups exposed to the tone $5 \mathrm{~s}$ before the gate opening had significantly longer latencies to leave the nest than the O-S group (left panel, Kruskal-Wallis, $\mathrm{H}=$ 18.6, $p<0.001$; pairwise comparisons, $p=0.008$ for T-S vs. O-S, $p=0.011$ for O-S vs. T-O, $p<0.001$ for O-S vs. T/O-S, $p=0.69$ for T-S vs. T-O, $p=0.631$ for T-S vs. T/O-S, $p=0.343$ for T/O-S vs. T-O). Once outside the nest, however, the latency to breach the trigger zone, enroute to the pellet, was not reliably different among the groups (Kruskal-Wallis, $\mathrm{H}=7.453, p=0.059$ ). In response to the triggered shock, owl or owl-shock, all groups showed similar escape-to-nest latencies (Kruskal-Wallis, $\mathrm{H}=6.141, p=0.105$ ).

(C) Representative track plot examples from T-S, O-S, T/O-S and T-O animals during the baseline, when 
478 animals successfully procured the pellet, and during the fear conditioning, when the same animals fled

479 from shock, owl or owl-shock stimuli and thus unable to attain the pellet. (D) Mean instantaneous speed

$480( \pm$ SEM) of each group $2 \mathrm{sec}$ before and after the shock, owl or owl-shock onset $(t=0)$. Thin, grey lines

481 represent individual animal data. (E) All groups showed comparable escape speed to the shock, owl, and

482 owl-shock stimuli (Kruskal-Wallis, $\mathrm{H}=0.901, p=0.825)$. (F) Representative track plots showing escape

483 paths of T-S, O-S, T/O-S and T-O animals. The inset silhouette images show that the T-S and T-O

484 animals were facing forward at the time of the shock or owl stimulus whereas the O-S and T/O-S animals

485 were turning back at the time of the shock stimulus because of the $100 \mathrm{~ms}$ owl-shock interstimulus

486 interval. (G) Mean escape distance ( \pm SEM) from the trigger zone to the nest. The O-S and T/O-S groups

487 travelled longer distances to escape compared to the T-S and T-O groups (Kruskal-Wallis, $\mathrm{H}=21.98, p<$

4880.001 ; pairwise comparisons, $p=0.014$ for T-S vs. T/O-S, $p=0.008$ for T/O-S vs T-O, $p=0.001$ for T-S

489 vs. O-S, $p=0.001$ for O-S vs T-O). (H) Representative vector plots of each group showing variabilities in

490 their escape paths. (I) Mean variance $( \pm$ SEM) of escape trajectory angles (radian) from the trigger zone

491 to the nest. The O-S and T/O-S groups had greater variance in their escape trajectories when fleeing

492 back to the nest (Kruskal-Wallis, $\mathrm{H}=22.37, p<0.001$; pairwise comparisons, $p=0.022$ for T-S vs. T/O-S,

$493 p=0.003$ for T/O-S vs T-O, $p=0.002$ for T-S vs. O-S, $p<0.001$ for O-S vs T-O). ( $\dagger$ compared to T-S,

$494 \mathrm{~T} / \mathrm{O}-\mathrm{S}$, and T-O; ${ }^{*}$ compared to O-S and T/O-S, $p<0.05,{ }^{* *} p<0.01,{ }^{* * *} p<0.001$; \# compared to T/O-S,

$495 \quad p<0.05, \# \#<0.01)$. 

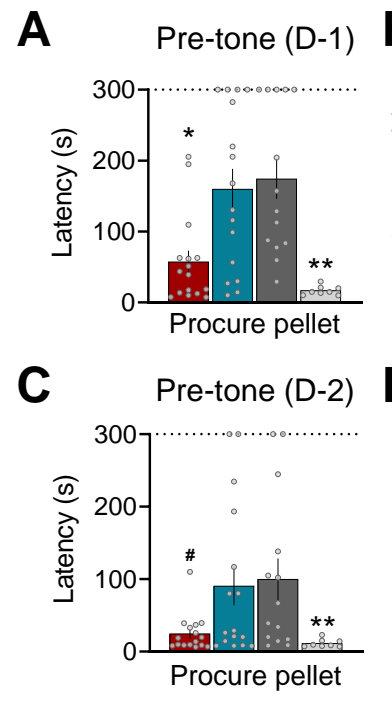

$\mathbf{E}$
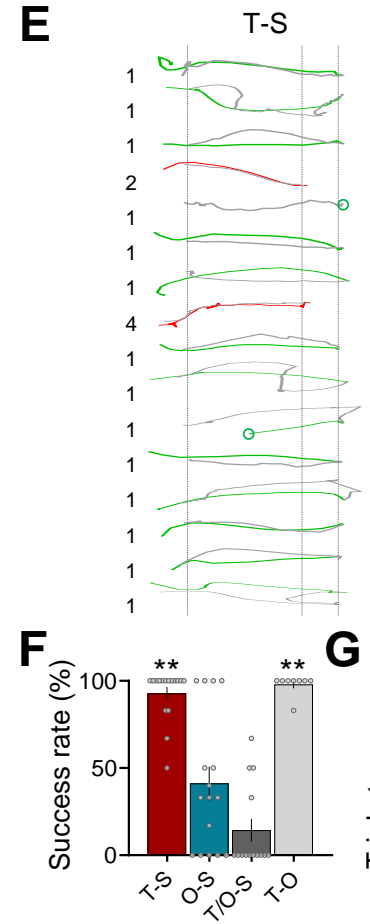

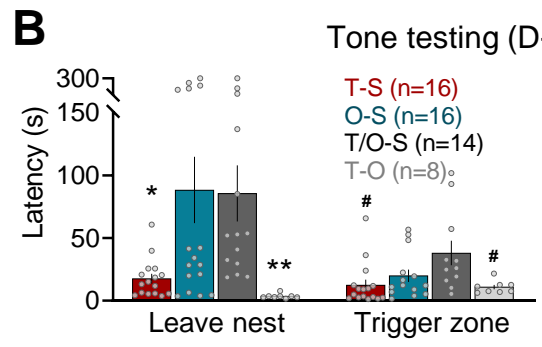

\section{D}

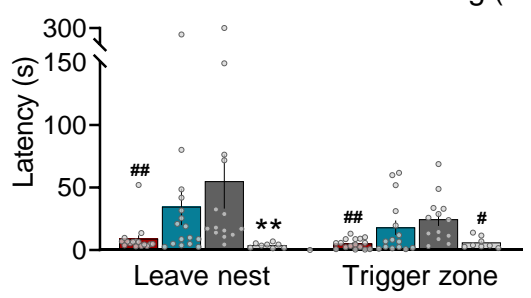

O-S

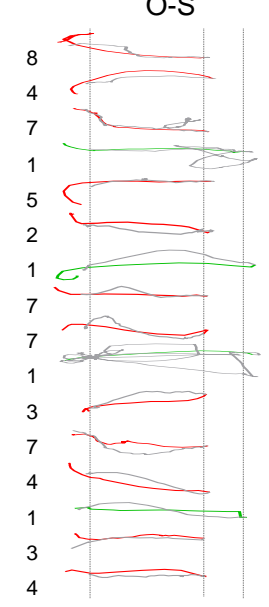

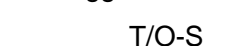

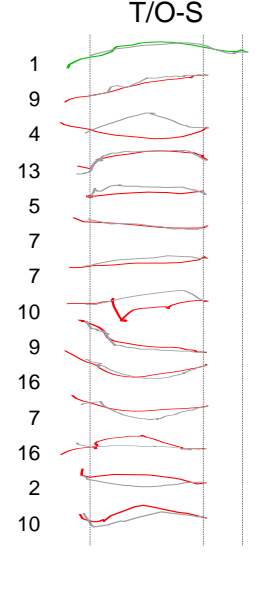

During tone $(60 \mathrm{~s})$

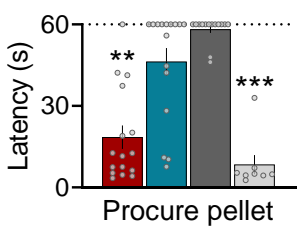

During tone (60 s)

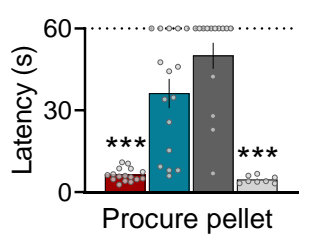

$\mathrm{T}-\mathrm{O}$

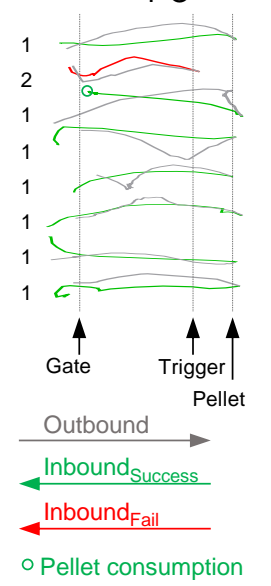

D-2

H D-1
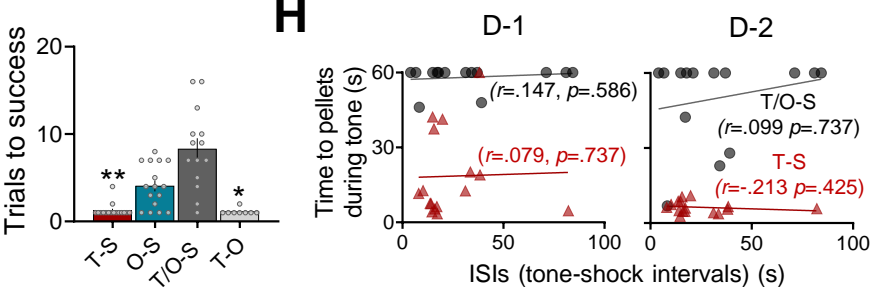

Fig. 3 Foraging and escape behaviors during tone testing. (A) The mean latency ( \pm SEM) to procure the pellet during the pre-tone baseline trials on testing day 1 (D-1). Both O-S and T/O-S groups took

503 significantly longer times to exit (gate opening, $t=0$ ) and return to the nest with the pellet than T-S and T-O

504 groups (Kruskal-Wallis, $\mathrm{H}=20.518, p<0.001$; pairwise comparisons, $P=0.003$ for T-S vs. T/O-S, $p<$

5050.001 for T/O-S vs. T-O, $p=0.013$ for T-S vs. O-S, $p<0.001$ for O-S vs. T-O). (B) The times (mean \pm

506 SEM) to leave nest and reach trigger zone on day 1 tone test trials. Both O-S and T/O-S groups had 
longer latencies to leave nest (Kruskal-Wallis, $\mathrm{H}=27.071, p<0.001$; pairwise comparisons, $p=0.003$ for T-S vs. T/O-S, $p<0.001$ for T/O-S vs. T-O, $p=0.044$ for T-S vs. O-S, $p<0.001$ for O-S vs. T-O. Once outside the nest, the T/O-S group took longer time to reach the trigger zone than the T-S and T-O (Kruskal-Wallis, $\mathrm{H}=9.153, p=0.027$; pairwise comparisons, $p=0.019$ for T-S vs. T/O-S, $p=0.042$ for T/O-S vs. T-O). During the tone test, the latencies to procure the pellet within the $60 \mathrm{~s}$ allotted time were significantly longer in O-S and T/O-S animals compared to T-S and T-O animals (Kruskal-Wallis, $\mathrm{H}=$ 34.428, $p<0.001$; pairwise comparisons, $p<0.001$ for T-S vs. T/O-S, $p<0.001$ for T/O-S vs. T-O, $p=$ 0.002 for T-S vs. O-S, $p<0.001$ for O-S vs. T-O). (C) The mean latency $( \pm$ SEM $)$ to procure the pellet during the pre-tone baseline trials on testing day 2 (D-2). O-S and T/O-S groups continued to have longer latencies to exit (gate opening, $\mathrm{t}=0$ ) and return to the nest with the pellet than $\mathrm{T}-\mathrm{S}$ and $\mathrm{T}-\mathrm{O}$ groups (Kruskal-Wallis, $\mathrm{H}=12.47, p=0.006$; pairwise comparisons, $p=0.022$ for T-S vs. T/O-S, $p=0.002$ for T/O-S vs. T-O, $P=0.009$ for O-S vs. T-O). (D) The times (mean \pm SEM) to leave nest and reach trigger zone on day 2 tone test trials. There were group differences in the latencies to leave nest (Kruskal-Wallis, $\mathrm{H}=21.505, p<0.001$; pairwise comparisons, $p=0.001$ for T-S vs. T/O-S, $p<0.001$ for T/O-S vs. T-O, $p$ $=0.002$ for O-S vs. T-O). Once outside the nest, there were group differences in the latencies to reach the trigger zone (Kruskal-Wallis, $\mathrm{H}=21.531, p<0.001$; pairwise comparisons, $p<0.001$ for T-S vs. T/O$\mathrm{S}, p<0.001$ for T/O-S vs. T-O, $p=0.037$ for $\mathrm{O}-\mathrm{S}$ vs. T-O). During the tone test, the latencies to procure the pellet within the $60 \mathrm{~s}$ allotted time were significantly longer in O-S and T/O-S animals compared to T-S and T-O animals (Kruskal-Wallis, $\mathrm{H}=37.223, p<0.001$; pairwise comparisons, $p<0.001$ for T-S vs. T/O$\mathrm{S}, p<0.001$ for T/O-S vs. T-O, $p<0.001$ for T-S vs. O-S, $p<0.001$ for O-S vs. T-O). (E) Individual track plots during the first tone exposure from all animals from each group. The parenthesized numbers next to plots represent the trial(s) needed for successful foraging. (F) The overall success rates of procuring the pellet on the first testing day were significantly lower in the O-S and T/O-S groups compared to the T-S and T-O groups (Kruskal-Wallis, $\mathrm{H}=32.299, p<0.001$; pairwise comparisons, $p<0.001$ for T-S vs. T/OS, $p<0.001$ for T/O-S vs. T-O, $p=0.001$ for T-S vs. O-S, $p=0.003$ for O-S vs. T-O). (G) The O-S and T/O-S animals required extended trials to obtain the pellet (Kruskal-Wallis, $\mathrm{H}=32.004, p<0.001$; pairwise comparisons, $p<0.001$ for T-S vs. T/O-S, $p<0.001$ for T/O-S vs. T-O, $p=0.002$ for T-S vs. O$\mathrm{S}, p=0.011$ for O-S vs. T-O). (H) In T-S and T/O-S animals, there were no reliable correlations 
535 (Spearman's correlation coefficient) between the tone-induced suppression of pellet procurement (an

536 index of fear) and the temporal intervals (i.e., ISIs) between tone CS onset and shock US onset in neither

537 testing day 1 nor 2 . ( ${ }^{*}$ compared to both O-S and T/O-S, $p<0.05,{ }^{* *} p<0.01,{ }^{* * *} p<0.001$; \# compared

538 to T/O-S, $p<0.05, p<0.01)$.

539

540

541

542

543

544

545

546

547

548

549

550

551

552

553

554 
555 Supplementary Information for

556

557

558

559

560

561

562

563

564

565

566

567

568

569

570

571

572

573

574

575

576

577

578

579

580

581

582

583

584

585

586

587

588

589

590

591

\section{Pavlovian fear conditioning does not readily occur in rats in naturalistic} environments

Peter R. Zambetti ${ }^{1}$, Bryan P. Schuessler ${ }^{1}$, Bryce E. Lecamp², Andrew Shin ${ }^{3}$, Eun Joo Kim¹, and Jeansok

J. $\mathrm{Kim}^{1 *}$

${ }^{1}$ Department of Psychology, University of Washington, Seattle, WA 98195

2Undergraduate Program in Neuroscience, University of Washington, Seattle, WA 98195

3Undergraduate Program in Human Biology, Stanford University, Stanford, CA 94305

*Jeansok J. Kim

Email: jeansokk@u.washington.edu

This PDF file includes:

Figures S1 to S3

Legends for Movies S1 to S2

Other supplementary materials for this manuscript include the following:

Movies S1 to S2 
592

600

601

602

603

604

605

606

607

608

609

610

611

612

613

614

615

616

617

618

619

620

621

622

623

624

625

626

627

628

629

630

631

632

633

634

635

636

637

638

639

\section{Baseline acquisition}

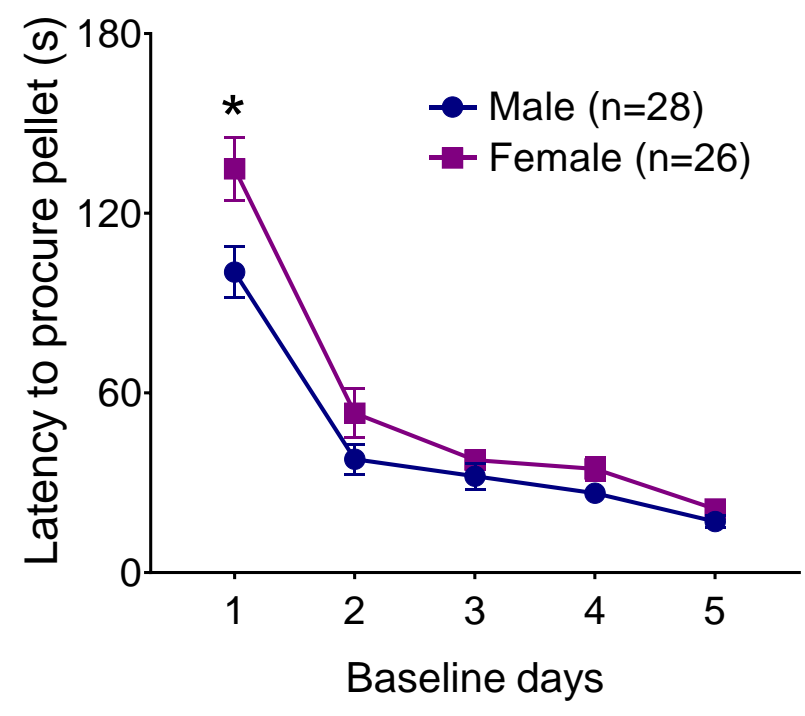

Fig. S1. Initial sex differences in the baseline latency to procure pellets. Average latencies $( \pm S E M)$

to procure food pellets in foraging area. Females had longer latencies to procure pellets than males during the first baseline session day 1 (Mann-Whitney $U, z=2.476, p=0.013$ ) but not subsequent baseline session days 2-5 (Mann-Whitney $U$, Baseline 2: $z=1.039, p=0.299$; Baseline 3: $z=1.922, p=$ 0.055; Baseline 4: $z=1.112, p=0.266$; Baseline $5: z=1.904, p=0.057) .{ }^{*} p<0.05$. 
640

641

642

643

644

645

646

647

648

649

650

651

652

653

654

655

656

657

658

659

660

661

662

663

664

665

666

667

668

669

670

671

672

673

674

675

676

677

678

679

680

681

682

683

684

685

686

687

688

689

A

\section{Tone test (D-1)}

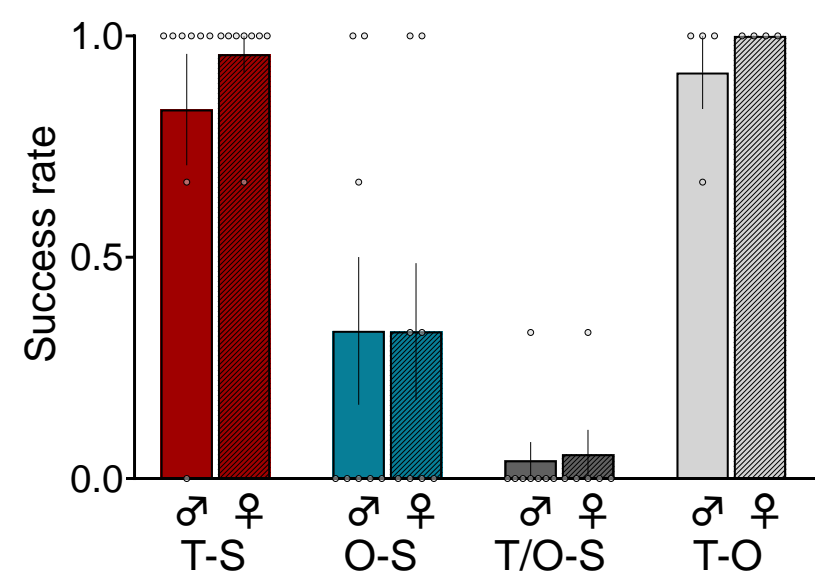

B

\section{Tone test (D-2)}

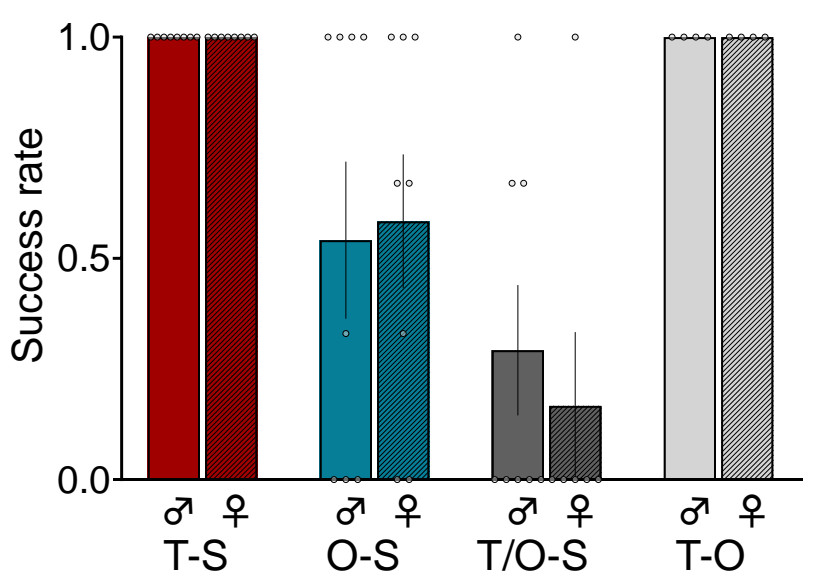

Fig. S2. No reliable sex differences in the procurement of pellets during tone tests. (A) Mean $( \pm$ SEM) success rate for procuring food pellets during the first day of tone testing. No significant differences were found between males and females in tone-shock (T-S), owl-shock (O-S), tone/owl-shock (T/O-S) and tone-owl (T-O) groups (Mann-Whitney U; $z=0.694, p=0.645$ for $\mathrm{T}-\mathrm{S} ; \mathrm{z}=1.0, p=0.317$ for O-S; $\mathrm{z}=$ $0.212, p=1.0$ for T/O-S; $z=0.234, p=0.815$ for $T-O)$. (B) Mean ( \pm SEM) success rate for procuring food 
bioRxiv preprint doi: https://doi.org/10.1101/2021.10.20.465116; this version posted October 21, 2021. The copyright holder for this preprint (which was not certified by peer review) is the author/funder, who has granted bioRxiv a license to display the preprint in perpetuity. It is made available under aCC-BY 4.0 International license.

690 pellets during the second day of tone testing. No sex differences were observed in all groups (Mann-

691 Whitney; $\mathrm{z}=0, p=1.0$ for $\mathrm{T}-\mathrm{S} ; \mathrm{z}=0.056, p=0.955$ for $\mathrm{O}-\mathrm{S} ; \mathrm{z}=-0.649, p=0.662$ for $\mathrm{T} / \mathrm{O}-\mathrm{S} ; \mathrm{z}=0, p=$ 6921.0 for T-O).

693 


\section{Pre-tone baselines}

695

696

697

698

699

700

701

702

703

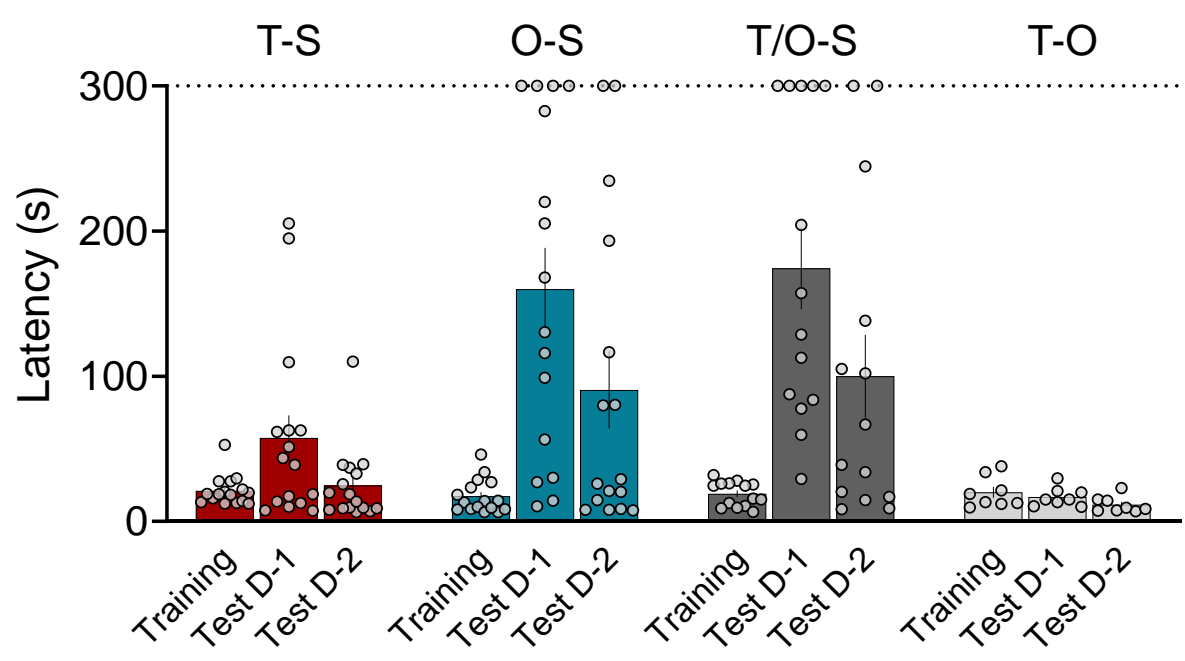

704

705

Fig. S3. Comparisons of latencies to procure pellets during pre-fear conditioning baseline and

706

pre-tone testing baseline days 1 and 2 . The baseline latencies to procure pellets prior to the fear

707 conditioning session (Fig. 2A) were not statistically different from the day 1 (Fig. 3A) and day 2 (Fig. 3C) pre-tone test baseline latencies after the fear conditioning session in both tone-shock (T-S) and tone-owl (T-O) paired animals (Related-samples Wilcoxon signed rank test; Baseline vs. D-1: $z=1.293, p=0.196$ for T-S; $z=-0.560, p=0.575$ for T-O; Baseline vs. $D-2: z=-0.155, p=0.877$ for T-S; $z=-1.82, p=0.069$ for T-O). This indicates that neither the tone-shock group nor the tone-owl group showed evidence of contextual fear conditioning. 


\section{Legends for supplementary movies}

723

724 Movie S1.

725 Representative foraging and escape behaviors of a rat presented with an owl-shock pairing. As the

726 animal come near a pellet, it encounters a swooping owl (from behind a black curtain) followed by a

727 dorsal neck/body shock pain. The rat flees to the nest without procuring the pellet.

728

729 Movie S2.

730 The next day, as the same O-S rat advances towards a pellet, a novel tone is presented for the first time.

731 In response to the tone, the rat promptly flees to the nest without procuring the pellet.

732

733

734

735 